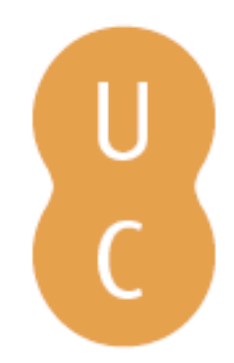

\title{
nombalina
}

Von der musikalischen farbe: der diskurs über chroma undcolor in mittelalter und renaissance
Autor(es):
Niemöller, Klaus Wolfgang
Publicado por: Imprensa da Universidade de Coimbra
URL
persistente:
URI:http://hdl.handle.net/10316.2/30034
DOI:
DOI:http://dx.doi.org/10.14195/978-989-26-0567-8_4
Accessed : $\quad$ 26-Apr-2023 12:59:45

A navegação consulta e descarregamento dos títulos inseridos nas Bibliotecas Digitais UC Digitalis, UC Pombalina e UC Impactum, pressupõem a aceitação plena e sem reservas dos Termos e Condições de Uso destas Bibliotecas Digitais, disponíveis em https://digitalis.uc.pt/pt-pt/termos.

Conforme exposto nos referidos Termos e Condições de Uso, o descarregamento de títulos de acesso restrito requer uma licença válida de autorização devendo o utilizador aceder ao(s) documento(s) a partir de um endereço de IP da instituição detentora da supramencionada licença.

Ao utilizador é apenas permitido o descarregamento para uso pessoal, pelo que o emprego do(s) título(s) descarregado(s) para outro fim, designadamente comercial, carece de autorização do respetivo autor ou editor da obra.

Na medida em que todas as obras da UC Digitalis se encontram protegidas pelo Código do Direito de Autor e Direitos Conexos e demais legislação aplicável, toda a cópia, parcial ou total, deste documento, nos casos em que é legalmente admitida, deverá conter ou fazer-se acompanhar por este aviso.

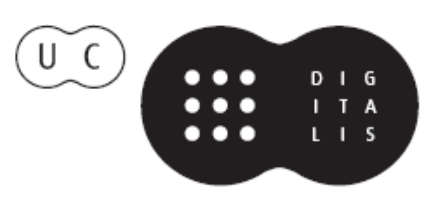




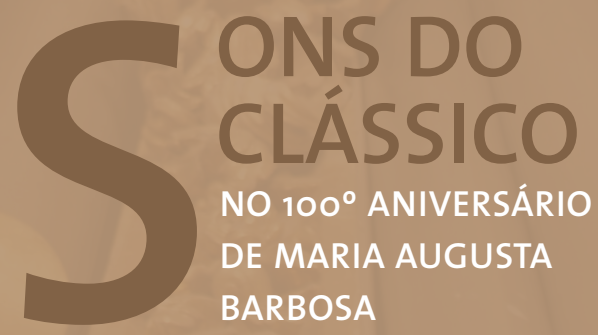

J. M. Pedrosa Cardoso

Margarida Lopes de Miranda COORDENAÇÃO 
Klaus Wolfgang Niemöller

Musikwissenschaftliches Institut - Universität zu Köln

\section{VON DER MUSIKALISCHEN FARBE. DER DISKURS ÜBER CHROMA UND COLOR IN MITTELALTER UND RENAISSANCE}

Seit der Antike bis zur Gegenwart wird eine Verbindung zwischen Musik und Farbe thematisiert. Dies geschieht unter vielfältigen Aspekten und keineswegs nur durch Musiker oder Musiktheoretiker. Während im 20. Jahrhundert die Bildende Kunst durch die Klangkunst inspiriert wurde, gingen umgekehrt in die Musik farbliche Vorstellungen von der „Tonmalerei“ bis zur „Klangfarbe“ ein. Die gegenseitigen metaphorischen Anleihen selbst im gegenwärtigen Sprachgebrauch reichen vom „schwarzen“ Bass und dem „Koloratur“-Sopran bis zum „Farbton“. Die Bereiche, in denen diese Konstellation diskutiert wurden und werden, reichen von der Naturwissenschaft bis zur Psychologie, von der Synästhesie bis zur Klangtechnik. ${ }^{3}$ Nachdem schon seit Carl Maria von Webers Freischütz und dann durch Hector Berlioz, Richard Wagner und Richard Strauss die Orchesterfarben in der Instrumentation als spezielle Klang- und Ausdrucksmittel entwickelt worden waren, erlangten im Expressionismus die synästhetischen Vorstellungen von Musik und Farbe einen Höhepunkt. ${ }^{4}$ Ein Meilenstein war Alexander Skrjabins symphonische Dichtung Prometheus. Le poème du feu, die 1911 in Moskau uraufgeführt wurde. Die Partitur sah neben dem Soloklavier ein „clavier à lumière“ vor, durch das die wechselnden Klangzentren des Quartenakkordes mit ihrem jeweiligen Farbenhintergrund auf eine Leinwand projeziert werden sollten. Angeregt worden war Skrjabin, der Farben mit ganz

3 K. Maurer (Hrsg.) 1985; H. de la Motte Haber (Hrsg.) 1996; M. Brzoska und E. Schmierer (Hrsg.) 1996.

4 B. Kienscherf 1996; J. Jewanski u. N. Stiller (Hrsg.) 2006. 
konkreten musikalischen Elementen verband, durch die Erfindung einer Farben-Orgel (colour organ) des englischen Kunstprofessors Wallace Rimingnton. Erst 1915 kam es in New York zu einer Realisierung der Farblichtprojektion. Arnold Schönberg - selbst expressionistischer Maler - hatte bereits 1908 dem II. Stück seiner Fünf Orchesterstücke op.16 die Überschrift Farben gegeben, eingelöst durch wechselnde Instrumentengruppen für die chromatischen Veränderungen des Grundakkordes, und 1911 dachte er in seiner Harmonielehre über die Möglichkeit einer „Klangfarbenmelodie“ nach. Gleichzeitig arbeitete er an dem Einakter Die glückliche Hand, in dem er parallel zu Wassily Kandinskys „Bühnenkomposition Der gelbe Klang systematisch Farbwirkungen von Bühne, Kostümen und Licht genau festlegte. Höhepunkt ist die Farbensymphonie zu der pantomimischen Darstellung, wie der Mann visionär die Hingabe des Weibes an den Herrn erlebt, zu der in einer Lichtpartitur die Farbveränderungen genau notiert sind. Das Faszinierende solcher Ideen durchdringt auch die Kompositionen von Olivier Messiaen und Karlheinz Stockhausen. Während sich in Messiaens Couleurs de la cité celeste die Orchesterfarben gemäss den Farbwandlungen entwickeln („les complexes de sons et timbres, évoluent à la facon des couleurs"), hat Stockhausen in seiner Oper Dienstag aus Licht für die Schlussszene elektronische Klänge auf Synthesizer, Sampler und Computer vorgesehen, mit denen die in der Partitur aufgelisteten 131 Klanfarben produziert werden.

Innerhalb der sehr unterschiedlichen Aspekte, die im Laufe der Jahrhunderte zu der Parallelisierung von Musik und Farbe führten, kommt der berühmten und aussergewöhnlichen Disputation zwischen Vincente Lusitano und Nicola Vicentino in Rom 1551 um die Chromatik besondere Bedeutung zu, und zwar einerseits durch den renaissancehaften Rückgriff auf die antike Musiktheorie, andererseits durch die zukunftsträchtige Anwendung auf eine konkrete Komposition. Anlass, Umstände und Inhalte der Disputation sind nach Henry William Kaufmanns Monographie über Nicola Vicentino (1966) umfassend von Maria Augusta Alves Barbosa in ihrer Kölner Dissertation von 1970 über Vincentius Lusitanus dargelegt worden. Um die in diesem Beitrag herauszuarbeitenden übergreifenden Perspektiven zu formulieren, genügt daher zunächst eine komprimierte Zusammenfassung als Basis.5 Ausgangspunkt

\footnotetext{
5 H. W. Kaufmann 1966; M. A. A. Barbosa 1970, besonders S. 196f. u. 236 f.
} 
war die Aufführung eines Regina caeli in einer privaten Akademie, dessen tonale Disposition Lusitano nach der Darstellung Vicentinos in seinem Traktat L'antica musica ridotta alla moderna practica 1555 als rein diatonisch beurteilte, während Vicentino behauptete, in der Musik der Gegenwart seien das chromatische und enharmonische Tongeschlecht mit dem diatonischen vermischt. Nach mehreren Diskussionsrunden sind die schriftlichen Stellungnahmen entscheidend, die beide Kontrahenten an den zum Schiedsrichter bestellten Ghiselin Danckerts sandten, als dieser zeitweilig abwesend war. Lusitano beruft sich nun auf die Unterscheidung der drei antiken Tongeschlechter (genera) und die jeweilige Intervallkonstellation innerhalb des Tetrachords auf Boethius' De institutione musica ${ }^{6}$, den er wörtlich zitiert. Damit beruft er sich nicht nur auf eine musiktheoretische Autorität, die das ganze lateinische Mittelalter herrschte, sondern auch den Autor, der ihr die antike Musiktheorie vermittelte. Dazu gehört auch die hier relevante Lehre von den drei Tongeschlechtern, die auf der pythagoräischen Methode beruht, die Intervalle durch Zahlenverhältnisse zu definieren. ${ }^{7}$ Boethius übersetzt in dem von Lusitano herangezogenen Zitat das zugrunde liegende griechische Wort chroma (Farbe) ins lateinische color und erläutert gleichzeitig, was dies in der Musik meint und aus welcher Vorstellung heraus die Vokabel „Farbe“ auf musikalische Phänomene übertragen wurde:

\begin{abstract}
Chroma autem quod dicitur color quasi iam ab huiusmodi intentione prima [tetrachordum diatonicum], mutatio. Cantaturper Semitonium et semitonium et tria Semitonia: tota enim Diatesseron consonantia et duorum tonorum ac semitonij, sed non pleni. Tractum est autem hoc vocabulum ut diceretur chroma a superficiebus que cum permutantur, in alium transeunt colorem.
\end{abstract}

Chroma aber, das color genannt wird, [ist] jetzt gleichsam eine Veränderung von der eben bestimmten ersten Anordnung [der des diatonischen Tetrachords]. Es wird gesungen durch Halbton und Halbton und drei Halbtöne: die gesamte Konsonanz der Quarte besteht deshalb aus zwei Ganztönen und einem Halbton, jedoch einem unvollkommenen. Hergeleitet ist aber die Vokabel chroma wie gesagt wird von Oberflächen, die, wenn sie verändert werden, in eine andere Farbe übergehen.

Im letzten Satz wird auf die Herkunft der doch eigentlich erstaunlichen Parallelisierung von Musik und Farbe, hingewiesen, die bis in die Antike zurückreicht. ${ }^{8}$ Chroma ist auf Musikalisches bezogen zwar nur eine Metapher,

\footnotetext{
6 Boethius 1966, S. 318.

7 B. Münxelhaus 1976

8 E. Roch 2010
} 
jedoch nimmt sie schon sehr früh Bezug auf die Wahrnehmung und die die psychische Wirkung der Musik, denn das griechische chroma bezog sich ethymologisch - primär auf die Hautfarbe. Die Veränderung der Hautfarbe durch Krankheit oder Emotionen wie Angst oder Zorn verband sich musikalisch mit der ethischen Bewertung der drei antiken Tongeschlechter. Etwa seit 400 v. Chr. wurden die Melodiegattungen des Diatonischen, des Chromatischen und des Enharmonischen unterschieden und dann auch ethischen Kriterien unterzogen, z. B. durch Plato. Das Chromatische mit seinen Halbtönen galt als symptomatisch für einen weichen, schwachen, unmännlichen Charakter, und damit geradezu als „ungriechsich“. Eine chromatisch geprägte Verzierungspraxis von Spielern der Kithara, die dazu die Zahl der Saiten erhöhten, wurde als unangemessen etwa für den musikalischen Vortrag der alten Mythen erachtet. Als männlich galt der dorische Melodie-Dialekt. Die den Intervallbestimmungen in der Skala des Tonsystems zugrundeliegenden Zahlenverhältnisse bestimmten also auch die Bewertung der darauf basierenden Musik. Von daher wird auch verständlich, dass bereits Aristoteles in seiner Schrift De sensu et sensibili von der Idee geleitet wurde, Farbzusammenstellungen auf dieselben einfachen Zahlenproportionen zurück zu führen wie musikalische Intervalle.

Mit dem Zitat von Boethius schliesst Lusitano die sich daran anschliessende musiktheoretische Tradierung der Begriffsfelder chroma und color ein. Dazu gehört auch das im Streit von Lusitano mit Vicentino angesprochene Grundproblem: das Verhältnis zwischen zahlenhaft-systematischer Ordnung von Musik in durch Proportionen bestimmbaren Intervallen und dem sinnlich wahrnehmbaren Klangeindruck, zwischen ratio, verankert in den Zahlenwissenschaften des Quadriviums, und sensus. In den mittelalterlichen Schriften zur Musiktheorie werden daher die beiden Termini auf verschiedene Bedeutungszusammenhänge bezogen, ${ }^{10}$ die zwischen „Chromatik“ und den tatsächlichen „Farben“ in der musikalischen Notation angesiedelt sind. Bemerkenswert ist, wie Guido von Arezzo um 1025 in seinem richtungweisenden Micrologus die Farbvorstellung auf die Kirchentonarten überträgt, die den Kirchengesängen der Gregorianik auf wundersame Weise die Erscheinung einer Farbe (coloris

\footnotetext{
9 F. Hentschel 2000.

10 Lexicon 2001, 5. Faszikel, Sp. 484-495 und 550-556.
} 
facies) zu verleihen schienen. ${ }^{11}$ Bereits 1480 hatte der italienische Musiktheoretiker Gaffurio in seinem gedruckten Theoricum opus musice discipline mit einem Kapitel „De tribus tetrachordum generibus“ (lib.V, cap. 2) die antike Lehre von den antiken Tongeschlechtern wieder aufgegriffen. ${ }^{12}$

Zunächst aber erscheint der Begriff „color“ in musikalischem Zusammenhang ganz konkret in der Notation. Die Färbung einer oder zwei Notenlinien (F-Linie: rot) in der Guidonischen Notation diente zur Anzeige einer bestimmten Tonhöhe in den gregorianischen Gesängen, ehe diese Aufgabe die Schlüssel übernahmen. Neben der Tonhöhe diente die Färbung in der Polyphonie auch zur Veränderung der rhythmischen Zeitstruktur in den Mensuren. Seit der Ars nova wurden die normalen schwarzen Noten zur Anzeige eines Mensurwechsels rot gefärbt. ${ }^{13}$ Insofern gilt auch hier die Bestimmung von color durch Boethius als mutatio. Der Begriff color nahm aber in der musikalischen Zeitstruktur der Hauptgattung der Ars nova, der isorhythmischen Motette, noch eine weitere, formbildende Bedeutung an: color als Wiederholung desselben Melodieabschnittes des cantus firmus im Tenor der Motette. Während Johannes de Muris in seinem Kapitel „De colore“ durchaus die Unterscheidung zwischen Wiederholung der Tonfolge (voces) und Wiederholung der rhythmischen Notenwerte (figurae) als talea unterscheidet, ${ }^{14}$ differenziert Johannes de Beldemandis in seinem längeren Kapitel „De colore et talea“, dass die Wiederholung von Tonfolge und Notenwerten nicht zugleich erfolgen können. ${ }^{15}$ Offensichtlich wird hier eine erweiterte Bedeutung von color herangezogen, als Färbung im Sinne von Ausschmücken. So formuliert dann Johannes de Garlandia: „Color ist die Schönheit des Klanges bzw. das Objekt beim Hören, wodurch das Gehör das Angenehme aufnimmt“, hervorgerufen

\footnotetext{
11 ...ut mirum in modum quandam ab ea coloris faciem ducere videantur. Micrologus 1955, S. 11

12 F. Remp 1989

13 W. Apel 1962, S. 134.

14 J. de Muris 58: Color in musica vocatur similitude figurarum unius processus pluries repetita positio in eodem cantus. Pro quo notandum quod nonnulli cantores ponunt diversitatem inter colorem et taleam. Nam vocant colorem, quando repetuntur eae voces; taleam vero quando repetuntur similes figurae.

15 P. de Beldemandis CS III, 226: vult namque ista [modernorum] opinion quod differentia sit inter colorem et taleam [...] assignando, quod in colore fiat repetitio similium vocum [...] et quod in talea fiat repetitio solum similium figurarum.
} 
durch eine formelhafte melodische Motivik, oder durch die Anreicherung des Klanges durch Mehrstimmigkeit wie im Conductus, oder durch melodische Wiederholungen wie in Rondelli und Volksgesängen. ${ }^{16}$ Johannes de Garlandia erlaubt die Beimischung von Dissonanzen nicht nur gemäss dem Kriterium der Schönheit (propter pulchritudinem), sondern auch „um des ,Schmucks' (propter colorem) willen. ${ }^{\text {"17 }}$ Die Beimischung von Dissonanzen wird sogar im wörtlichen Sinne „Farbe genannt“ und zwar im direkten Vergleich mit dem Maler, der den einfachen Farben unzählige sanfte Farbarten beimischt. ${ }^{18}$

Wesentlicher als der Vergleich mit der Malerei und ihren verschiednen Farben ist die Übernahme der Terminologie aus der Sprachdisziplin der Rhetorik, der color rbetoricus, wie Marchettus von Padua explizit formuliert, indem er speziell auf ihre Sinnfiguren verweist. ${ }^{19}$ Nach Cicero, der von einer durch Farben gemalten Rede spricht (coloribus picta ... oratio), werden in der mittelalterlichen Rhetorik zahlreiche Stilfiguren als Ausschmückungen (exornationes) aufgelistet, darunter „Wiederholungen“ (repetitiones) als Wortfigur. ${ }^{20}$ Damit kommen für die Musik gleich zwei Wesensseiten von color ins Blickfeld, das „Ausschmücken“ und die rhetorische Wirkungsabsicht. Diese affektive Wirkungsabsicht über Farbe in der Musik konkretisierte sich z.B. in Form von sogenannter „Augenmusik“ in der Schwärzung von an sich weissen Noten zum Zeichen der Trauer. So ist die berühmte Trauermusik von Josquin für seinen Lehrer (1495) Déploration sur la mort de Johannes Ockeghem mit dem Introitus der Totenmesse im Tenor symbolhaft nur in schwarzen Noten notiert. Das gewählte Notierungssystem hat ausserdem zur Folge, dass die Musik angemessen langsam und getragen ist. Ausschmücken, Verzieren ist bereits für die Vokalmusik als Umspielen einer Kernmelodik, als „Kolorierung“ musikgeschichtlich diskutiert worden, noch deutlicher wird das colorare aber in der Instrumentalmusik. Der Konstanzer Organist Hans

16 J. de Garlandia 1972, S. 95: Color est pulchritudo soni vel obiectum auditus, per quod auditus sucipit placventiam. Et fit multis modis: aut sono ordinate, aut in florificatione soni, aut in repetitione ejusdem vocis vel diverse.

17 Hentschel (wie Anmerkung 7), S. 181-184.

18 Sed et pictor naturales colores artificio transferens, innumera coloris genera placabilia simplicibus commiscet.

19 M. de Padua 1961, S. 71: in musica fiunt interdum colores ad pulchritudinem consonantiarum, sicut in grammatica fiunt colores rhetorici ad pulchritudinem sententiarum.

20 F. Quadlbauer 2003, Sp. 61f. 
Buchner hat bereits um 1530 in seinem Lehrbuch der Orgelkunst (fundamentum) gezeigt, wie die Choralmelodie des Te Deum laudamus nicht nur zu einem einfachen dreistimmigen Orgelsatz verwandelt wird, sondern auch in einem dritten Notenbeispiel, wie die Organisten jede Stimme mit ihren Kolorierungen versehen (organistae addunt cuique voci suos colores). ${ }^{21}$ So wurden wegen der gehäuften Verzierungen die deutschen Orgelkomponisten ab 1570 wohl auch zu einer Gruppe der „Koloristen“ zusammengefasst. ${ }^{22}$ In den Tabulaturbüchern von Elias Nicolaus Amerbach (1571) und Jacob Paix (1583) gehörte es zum Wesen der Intavolierung, dass sie „mit Fleiss coloriret“.

Die Einrichtung der Claviatur bei den Tasteninstrumenten mit schwarzen Obertasten für Halbtöne führt zu einer Auffassung von Chromatik“, die zu dem Disput Lusitano Vicentino zurückführt, ja bis zu Boethius. Sebastian Virdung hat 1511 in seiner Musica getutscht, der für Instrumentisten eingedeutschten lateinischen Musiklehre, im Dialog mit seinem Schüler darauf hingewiesen, dass nach Guido von Arezzo einige kamen, „die haben das [System mit 20 diatonischen Tonstufen] noch subtiler gemacht / und Boetium auch gelesen und nach dem andern geschlecht Cromaticum genant//das moncordum aufgeteilet." ${ }^{23}$ Bei der Frage, was denn das chromaticum genus sei, übersetzt Virdung nicht nur die Definition einer „Färbung“ von Boethius, sondern weist auch auf die zwei erweiterten Aspekte hin: die musikalische Verzierung und die chromatisch erweitertet Claviatur des Tasteninstruments, im Wortlaut: „Croma ist eben so vil gesagt als eyn farb / vnnd Cromaticum eben als eyn geferbt sind / Das heist man auch sunst zuzeiten etwas aussgestrichens oder ein wol geziertes ding / Also ist auch hie dye kunst Musica und das instrument noch vil subtiler aussgestrichen / vnd bas geziert /durch die semitonia des geschlechtes Cromaticum genent.“ Im diatonischen Tongeschlecht ergeben sich durch die Quarten mit zwei Ganztönen und einem kleinen Halbton 20 Tonstufen, „Stimmen“ (voces) oder „schlüssel“ (claves); dazu kommt die Dopppelstufe „bfahmi“. Die abgebildete Claviatur zählt so 22 Tasten (darunter zwei schwarze). In Analogie zu der chromatischen Doppelstufe bfahmi werden nun nach Virdung weitere 13 Halbtöne („myndere semitonia“)

\footnotetext{
21 H. Buchner 1974, S. $22 \mathrm{f}$.

22 A. Edler 1997, 27.

23 S. Virdung 1970, Bl. Eiij verso s.
} 
hinzugenommen, so dass sich „yetz gemainlich [aus] baider geschlecht“ für das Gesamtsystem über drei Oktaven 38 „schlüssel“ (claves) und damit 38 Tasten ergeben, die in einer zweiten Abbildung dargestellt bereits die moderne Tastatur aufweisen. Die weitere Entwicklung der Clavierinstrumente und ihrer Stimmungen führt wieder zu den zwischen Lusitano und Vicentino strittigen Grundfragen von chroma und color zurück. ${ }^{24}$

$\mathrm{Zu}$ Virdungs Zeit wurden also bereits dem achtstufigen Tonsystem chromatische Zwischentöne hinzugefügt, zunächst mit cis und es, fis und as. Dies zeigt das Diagramm des praktischen Tonsystems mit den Hexachorden der Solmisation, das von Johannes Cochlaeus 1512 in seinem Tetrachordum musices unter der Überschrift Diatonicum Guidonis introductorium abgebildet ist. In der scala ficta wird so schliesslich jedem Tonbuchstaben entweder ein b rotundum oder h quadratum hinzugefügt, ${ }^{25}$ wie bereits Andreas Ornitoparch 1517 schrieb. ${ }^{26}$ Er weist aber auch auf den Begriff hin, der verdeutlicht, dass die chromatischen Töne in der musica ficta keine selbständigen Tonstufen im diatonischen System darstellen, sondern als coniunctae nur eine improvisierte Veränderung von Ganzton in Halbton oder umgekehrt darstellen. ${ }^{27}$ Bereits im Traktat des Johannes Hollandrinus und seinen Tradierungen im 15. Jahrhundert wird den „coniunctae“ ein eigenes Kapitel gewidmet. Der systematische Nachweis von Halbtönen ausserhalb der Guidonischen Skala in den Gregorianischen Gesängen und die Bezeichnung als „coniunctae“ ist eine Besonderheit dieser Lehrtradition. ${ }^{28}$ Die Erniedrigung durch b-fa oder Erhöhung durch h-mi ist also mit der eigentlichen Tonstufe „verbunden“. Praktiziert wird sie sowohl durch die Singstimme wie auf dem Instrument (quod coniuncta secundum vocem hominis vel instrumenti est facere...). Ausdrücklich hebt Hollandrinus hervor, dass dies nicht als eine Veränderung (mutatio) eines Halbtones in einen Ganzton und umgekehrt zu verstehen ist, sondern dass nur an dem Tonort, an dem ein Ganzton bzw. Halbton zu sein

24 F. J. Ratte 1991.

25 H. von Loesch, 2003, S. 134, Abb. 3 u. S. 161-166.

26 A. Ornitoparch 1977, fol. Cv verso: Musica ficta fingit in quacunque clave quamcunque vocem.

${ }^{27}$ Est autem coniuncta, canere vocem in clave, que non est in ea. Uel est toni in semitonium aut semitonij in tonem subita et improuisa mutatio. (Ebenda).

28 Traditio 2010, Bd. I, S. 140 f. 
pflegt, ein anderer gesungen oder gespielt wird. ${ }^{29}$ Damit bezieht er sich auf Johannes de Muris, der eine Veränderung ausserhalb des regulären Systems der musica plana als irregulär erachtete (Vocatur autem irregularis mutatio falsa mutatio), jedoch nicht in der musica mensurabilis, weshalb bei einigen Instrumenten wie den Orgeln überall der Ganzton geteilt wird, um Konsonanzen im Zusammenklang herzustellen. ${ }^{30}$

Indem Franciscus Gafurius die halbtönigen Teilungen der Tonstufen als Fictas autem seu coloratas contrapuncti species, also die musica ficta nicht als musica falsa begreift, sondern als eine „Einfärbung“, verweist er darauf, dass diese „im Rahmen einer chromatischen Ausmessung ,kolorierte’ Gesänge zeigen, die auch als ficta bezeichnet werden (hae enim, quod cromatica dimensione ducuntur, coloratas demonstrant cantilenas, quas et fictas dicunt).

Nachdem bereits Gioseffo Zarlino sich 1548 ein chromatisches Clavicembalo hatte bauen lassen, präsentierte Vicentino in seiner Schrift L'antica musica 1555 sozusagen als Rechtfertigung für seine gegen Lusitano erlittene Niederlage sein enharmonisches Archicembalo. ${ }^{31}$ Im Anschluss an diese Instrumente konnte auch die Vision einer engen Verbindung von Farbe und Musik um 1590 durch die Konstruktion eines entsprechenden Instrumentes praktisch umgesetzt werden. Der Mailänder Maler Giuseppe Arcimboldo, Hofmaler von Kaiser Rudolf II. in Prag, noch heute berühmt durch seine Porträts aus Blumen und Früchten, war wohl der erste, der die Korrespondenz von Farben und Tönen auf einem chromatischen „gravicembalo“ darstellen liess. Der kaiserliche Musiker Mauro Cremonense sollte Arcimboldis „colori sopra una carta“ als Klänge auf dem Instrument darstellen. ${ }^{32}$ Vicentinus Gravicembalo, das sich am Hofe der d'Este in Ferrara befand, war dann wohl auch Anregung für die kühnen chromatischen Kompositionen von Carlo Gesualdo, der sich nach seiner Heirat mit Eleonore d'Este seit 1594 zwei Jahre

\footnotetext{
29 Ebenda Bd. II (2010, Bd.20), S.221 u. Bd. III (2011, Bd.21), S.77: Sequitur de naturis coniunctarum: Et non est intelligendum, quod tonus mutetur in semitonium, sed in loco in quo solebat esse tonus per coniunctam fit semitonium e econverso.

30 J. de Muris II, S.294: Ille igitur etsi dicantur falsa, quantum ad planam musicam, non tamen quantum ad musicam mensurabilem. Ideo in aliquibus instrumentis, ut in organis quasi ubique tonus dividitur in duo semitonia inaequalia, ut plures ibi fieri possunt concordie.

31 Ratte (wie Anm. 22), S. 385 - 396.

32 P. Preiss 1972, S. 163ff.
} 
in Ferrara aufhielt. Ein Madrigal wie „Moro lasso“ setzte mit der Chromatik zu diesem Beginn die neue ästhetische Richtung der affektiven Nachahmung der Textinhalte um. Sie hatte u.a. Vicentino gefordert, denn die Musik sei zu nichts anderem gemacht, „per esprimere il concetto, et le passioni et gli effetti di quelle“, wobei die die Intervalle der drei antiken Tongeschlechter „secondo il sogetto della parole" affektiv zu gebrauchen sind. ${ }^{33}$

Die zunächst in langen musiktheoretischen Diskursen aufgekommenen Begriffe von Farblichkeit in der Musik, chroma und color, haben so seit der Spätrenaissance unmittelbar in Kompositionspraxis und Aufführungsweise Einzug gehalten und im Barock eine Blüte erlebt, die bei Johann Sebastian Bach kulminiert, sei es im chromatischen Quartgang der rhetorischen Figur des passus duriusculus (Kantate „Weinen, Klagen, Sorgen“), sei es instrumental in der Chromatischen Fantasie und Fuge. Nur der in Rom als Jesuit wirkende Universalgelehrte Athanasius Kircher hat 1650 in seiner Musurgia universalis die ursprüngliche konkrete Verbindung zwischen Musik und Farbe wieder aufgegriffen. In einer Tabelle hat er 13 Intervalle innerhalb der Oktave, die als „grün“ die Mitte einnimmt (VIRIDIS DIAPASON) aufgeführt und sie mit bestimmten Farben in Relation gesetzt. Mit Blick auf die vorstehenden Überlegungen, die von Lusitanos und Vicentinos Kontroverse ausgingen, bilden so nicht von ungefähr den Rahmen der Tabelle der „weisse“ Halbton (Albus Semitonium) und der „schwarze“ Ganzton (Niger Tonus maior). ${ }^{34}$

\section{Bibliographie}

Apel, Willi (1962), Die Notation der polyphonen Musik 900-1600. Leipzig.

Barbosa, Maria Augusta Alves (1977), Vincentius Lusitanus. Ein portugiesischer Komponist und Musiktheoretiker des 16. Jahrhunderts. Lissabon.

Beldemandis, Prosdocimus de: Tractatus practice de musica mensurabili, CS III, 226 Boethius (1966), De institutione musica. Ed. Nachdruck Frankfurt.

Brzoska, Matthias und Schmierer, Elisabeth (Hrsg. 1996), Töne - Farben - Formen. Über Musik und die Bildenden Künste. Laaber.

33 K. W. Niemöller 2002, s.90f.

34 A. Kircher 1970, S. 568. 
Buchner, Hans (1974), Sämtliche Orgelwerke. Erster Teil: Fundamentum, hrsg. Jost Harro Schmidt. Frankfurt 1974.

Edler, Arnfried: Gattungen der Musikfür Tasteninstrumente., Teil 1: Von den Anfängen bis 1750, Laaber 1997, 27. (Handbuch der musikalischen Gattungen 7,1).

Garlandia, Johannes de (1972), De mensurabili musica. Ed. Erich Reimers, Wiesbaden.

Guido Aretinus, Micrologus (1955), ed. Joseph Smits van Waesberghe.

Haber, Helga De la Motte (Hrsg. 1996), Musik und bildende Kunst. Von der Tonmalerei zur Klangstruktur. Laaber.

Hentschel, Frank (2000), Sinnlichkeit und Vernunft in der mittelalterlichen Musiktheorie. Stuttgart.

Jewanski, Jörg u. Stiller, Natalie (Hrsg. 2006), Farbe - Licht-Musik. Synästhesie und Farblichtmusik. Frankfurt.

Kaufmann, Henry William (1966), The Life and Works of Nicola Vicentino (1511 - c. 1570). American Institute of Musicology.

Kienscherf, Barbara (1996), Das Auge hört mit. Die Idee der Farblichtmusik und ibre Problematik - beispielhaft dargestellt an Werken von Alexander Skrjabin und Arnold Schönberg. Laaber.

Kircher, Athanasius (1970), Musurgia universalis. Rom 1650, Nachdruck Hildesheim.

Lexicon Musicum Latinum Medii Aevi. Wörterbuch der lateinischen Musikterminologie des Mittelalters bis zum Ausgang des 15. Jabrhunderts (2001) hrsg. Michael Bernhard. München.

Loesch, Heinz von (2003), „Musica - Musica practica - Musica poetica“, in: Deutsche Musiktheorie des 15. bis 17. Jahrhunderts. Darmstadt.

Maurer, Karin (Hrsg. 1985), Vom Klang der Bilder. Die Musik in der Bildenden Kunst des 20. Jahrhunderts. München.

Münxelhaus, Barbara (1976), Pythagoras musicus. Zur Rezeption der pythagoräischen Musiktheorie als quadrivialer Wissenschaft im lateinischen Mittelalter. Bonn-Bad Godesberg.

Muris, Johannes de, Libellus cantus mensurabilis, Coussemaker, Scriptores de musica (CS).

Muris, Johannes de, Speculum musicae, Gerbert, Scriptores de musica (GS) II, S.294

Niemöller, Klaus Wolfgang (2002), „Tradition und Innovation des Affekt-Denkens im Musikschrifttum des 16. Jahrhunderts" in: Tugenden und Affekt e in der Philosophie, Literatur und Kunst der Renaissance. Hrsg. Joachim Poeschke u.a., Münster.

Ornitoparch,Andreas (1977), Musicae active micrologus. Leipzig 1517, Nachdruck Hildesheim.

Padua, Marchetus de (1961), Pomerium. Ed. Joseph Vecchi.

Preiss, Pavel (1972), „Farbe und Klang in der Theorie und Praxis des Manierismus“ in Colloquium Musica Bohemica et Europea Brno 1970. Brno. 
Quadlbauer F. (2003), „Colores rhetorici“ in: Lexikon des Mittelalters III. München.

Ratte, Franz Joseph (1991), Die Temperatur der Clavierinstrumente. Quellenstudien $z u$ den theoretischen Grundlagen und praktischen Anwendungen von der Antike bis ins 17. Jahrhundert. Kassel.

Remp, Frieder (1989), „Elementar - und Satzlehre von Tinctoris bis Zarlino“ in Italienische Musiktheorie im 16. und 17. Jahrhundert. Antikenrezeption und Satzlehre. Darmstadt.

Roch, Eckhard (2010), Chroma - Color - Farbe. Urtsprung und Funktion der Farbenmetapher in der antiklen Musiktheorie. Mainz.

Traditio Iohannis Hollandrini, Hrsg. Michael Bernhard u. Elzbieta Witkowska-Zaremba, 2010. Bd. I, München.

Virdung, Sebastian (1970), Musica getutscht 1511. Faksimile-Nachdruck hrsg. Klaus Wolfgang Niemöller, Kassel. 